\title{
TERT Promoter Mutation Predicts Radioiodine-Refractory Character in Distant Metastatic Differentiated Thyroid Cancer
}

\author{
Xue Yang ${ }^{1}$, Jiao $\mathrm{Li}^{2}$, Xiaoyi $\mathrm{Li}^{3}$, Zhiyong Liang ${ }^{4}$, Wen $\mathrm{Gao}^{2}$, Jun Liang ${ }^{5}$, Shujun Cheng*1, and Yansong Lin*2 \\ ${ }^{1}$ Department of Etiology and Carcinogenesis, National Cancer Center/Cancer Hospital, Chinese Academy of Medical Sciences and \\ Peking Union Medical College, Beijing, China; ${ }^{2}$ Department of Nuclear Medicine, Peking Union Medical College Hospital, Chinese \\ Academy of Medical Sciences and Peking Union Medical College, Beijing, China; ${ }^{3}$ Department of General Surgery, Peking Union \\ Medical College Hospital, Chinese Academy of Medical Sciences and Peking Union Medical College, Beijing, China; ${ }^{4}$ Department of \\ Pathology, Peking Union Medical College Hospital, Chinese Academy of Medical Sciences and Peking Union Medical College, \\ Beijing, China; and ${ }^{5}$ Department of Medical Oncology, Peking University Cancer Hospital and Institute, Beijing, China
}

Telomerase reverse transcriptase (TERT) promoter mutation has been reported to be associated with aggressive characteristics in differentiated thyroid cancer (DTC). This study examined the status of TERT mutation in distant metastatic DTC and evaluated the correlation between TERT mutation and radioiodine uptake, as well as that between TERT mutation and therapy response. Methods: TERT promoter and B-Raf proto-oncogene (BRAF) V600E mutation were retrospectively examined in primary tumors of 66 patients with distant metastatic DTC. Stimulated thyroglobulin (sTg) changes, radioiodine uptake status (avid or nonavid), and other imaging evidence were analyzed to evaluate therapy response. After a median follow-up of 46.5 mo (interquartile range, 29.0-70.5 mo), therapy response was classified as either disease control or refractory. Results: The prevalence of TERT mutations was $22.73 \%(15 / 66)$, of which C228T mutation was more prevalent (13/15) than C250T mutation (2/15). Rising sTg was noticed in $93.33 \%(14 / 15)$ of the TERT mutation group. Of cases negative for both mutations, $78.12 \%$ (25/ 32) presented with decreased sTg. TERT mutation closely correlated with a poor response to radioiodine therapy $(P<0.001)$, and all 15 patients were classified as refractory to radioiodine therapy, with a positive predictive value of $100 \%$ at the endpoint of follow-up. TERT mutation was associated with older mean age at diagnosis $(P<0.001)$, larger mean tumor diameter $(P=0.013)$, and greater likelihood of both BRAF mutation coexistence $(P=0.044)$ and radioiodine-refractory character $(P<0.001)$. In the 36 cases whose imaging results underwent semiquantitative analysis, TERT mutation significantly correlated with non-radioiodine avidity, with a much lower mean tumor-to-background ratio (obtained from postradioiodine whole-body scanning) than in TERT wild-type cases $(P<0.001)$. In addition, patients with distant metastatic DTC with TERT mutation were more likely to lose radioiodine avidity at the initial radioiodine therapy than were those with only $B R A F$ mutation (8/8 vs. $5 / 11$; Fisher exact test, $P=0.018$ ). Conclusion: TERT mutation closely associates with non-radioiodine avidity in distant metastatic DTC, and when compared with BRAF mutation, TERT

Received Jun. 24, 2016; revision accepted Jul. 19, 2016.

For correspondence contact: Yansong Lin, Department of Nuclear Medicine, Peking Union Medical College Hospital, Chinese Academy of Medical Sciences and Peking Union Medical College, No. 1 Shuaifuyuan, Wangfujing

St., Dongcheng District, 100730, Beijing, China.

E-mail: linys@pumch.cn

${ }^{*}$ Contributed equally to this work.

Published online Aug. 4, 2016.

COPYRIGHT (c) 2017 by the Society of Nuclear Medicine and Molecular Imaging. mutation manifested a greater negative influence on radioiodine uptake. TERT mutation could also be used as an early predictor of radioiodine-refractory cases.

Key Words: differentiated thyroid carcinoma; TERT mutation; radioactive iodine therapy; therapy response

J Nucl Med 2017; 58:258-265

DOI: 10.2967/jnumed.116.180240

A though the clinical outcome of differentiated thyroid cancer (DTC) is favorable, with the 10-y overall survival rate being around $90 \%$, it can be life-threatening when distant metastasis occurs $(2.2 \%-23 \%$ of DTC patients) $(1,2)$. Serial treatment with radioiodine can render a $10-y$ survival rate of $42 \%$, despite the fact that distant metastasis contributes most to death from DTC (3). The efficacy of radioiodine therapy depends largely on the ability of distant metastatic lesions to take up radioiodine, and the 10-y survival rate can be as high as $56 \%$ in patients with an intense radioiodine accumulation and as low as $10 \%$ in those who lose radioiodine avidity (3). Therefore, the radioiodine concentration pattern closely correlates with the radioiodine therapy response and clinical outcome. Early identification of non-radioiodine avidity would be helpful in avoiding unnecessary radioiodine therapy and allowing for timely adjustment to a subsequent feasible therapy. In a previous study, we used ${ }^{99 \mathrm{~m}} \mathrm{Tc}-$ 3PRGD2 imaging targeting integrin $\alpha_{v} \beta_{3}$ to trace radioiodinerefractory metastatic lesions; active angiogenesis in rapidly growing radioiodine-refractory foci could be detected (4). In another study, we found that the B-Raf proto-oncogene (BRAF) V600E mutation was associated with non-radioiodine avidity in distant metastatic DTC (5), implying that molecular characteristics may drive the early identification of non-radioiodine avidity. The telomerase reverse transcriptase (TERT) mutation has been shown to be an aggressive molecular marker for follicular cell-derived thyroid neoplasm (6). C228T and C250T - two prevalent hot spots, or positions where there are frequent mutations-may cause hyperactivity of TERT promoter and induce overproliferation and carcinogenesis $(7,8)$. It remains unclear whether TERT mutation is associated with radioiodine uptake status in distant metastatic lesions and radioiodine therapy response. 
The aim of the present study was to determine the prevalence and frequency of TERT mutation in Chinese patients with distant DTC metastases and to analyze the correlation between TERT mutation and radioiodine uptake status and therapy response in distant metastatic lesions.

\section{MATERIALS AND METHODS}

\section{Patients and Tissue Samples}

The study protocol was approved by the ethical board of the Chinese Academy of Medical Sciences and Peking Union Medical College Hospital, and all subjects gave written informed consent.

Sixty-six cases of distant metastatic DTC were retrospectively retrieved from Peking Union Medical College Hospital. The overall median follow-up time was $46.5 \mathrm{mo}$ (interquartile range, 29.0-70.5 mo) after the initial treatment. Between August 2008 and January 2016, the patients had been referred for at least 2 courses of radioiodine therapy after total thyroidectomy. Of the 66 patients, 36 underwent both surgeries and each course of subsequent radioiodine therapy at Peking Union Medical College Hospital; these intact data allowed us to apply further semiquantitative analysis.

\section{Imaging Analysis}

Distant metastases were identified by imaging examinations such as chest $\mathrm{CT},{ }^{18} \mathrm{~F}-\mathrm{FDG}$ PET/CT, bone scanning, diagnostic radioiodine wholebody scanning, and post-radioiodine therapy whole-body scanning, in combination with an elevated thyroglobulin level. Semiquantitative analysis of radioiodine uptake in metastatic foci was conducted by the same clinician using SPECT region-of-interest software. Briefly, regions were drawn separately around target lesions and normal frontal cranial bone, the mean count of each region was determined, and the tumor-to-background (T/B) ratio was obtained. To avoid interference from the thyroid remnant, we calculated two T/B ratios for each of the 36 patients, one after the first radioiodine therapy, when the thyroid remnant was present, and another after the subsequent radioiodine therapy, when the residual thyroid tissue had been completely ablated. The radioiodine uptake status was categorized as radioiodine-avid or non-radioiodine-avid according to the findings on whole-body scanning of distant metastatic lesions.

\section{Serologic Examination}

Serologic examinations, including thyroid-stimulating hormone, thyroglobulin, and antithyroglobulin antibody levels, were measured before radioiodine therapy and during follow-up. Patients positive for antithyroglobulin antibody were ruled out. Thyroglobulin and antithyroglobulin antibody levels were measured using an electrochemiluminescence immunoassay (Roche Diagnostics $\mathrm{GmbH}$ ), and the thyroid-stimulating hormone level was measured using a chemiluminescence immunoassay (Siemens Healthcare Diagnostics Inc.) in the same laboratory. The thyroglobulin assay had a functional sensitivity of $0.1 \mathrm{ng} / \mathrm{mL}$. Stimulated thyroglobulin (sTg) was used as a serologic marker to evaluate the response to radioiodine therapy. After at least 2 courses of radioiodine therapy, the serologic response was categorized as up $(>20 \%$ increase in sTg compared with first radioiodine therapy), down ( $>20 \%$ decrease), or stable $(-20 \%$ to $+20 \%)$. At the end of follow-up, the radioiodine therapy response was classified as disease control or refractory.

\section{Evaluation Criteria for Radioiodine-Refractory DTC}

On the basis of the 2015 American Thyroid Association management guidelines (9) in combination with the 2014 Chinese management guidelines on DTC treatment by radioiodine (10), radioiodine-refractory structurally evident DTC in patients with appropriate thyroid stimulating hormone stimulation and iodine preparation is defined in 4 ways: the metastatic tissue does not concentrate radioiodine even after successful remnant thyroid ablation; the tumor tissue loses the ability to concentrate radioiodine after previous evidence of radioiodine-avid disease (in the absence of stable iodine contamination); radioiodine is concentrated in some lesions but not in others; or metastatic disease progresses despite a significant concentration of radioiodine.

\section{DNA Isolation}

Genomic DNA was extracted from four 5- $\mu \mathrm{m}$-thick slices of formalinfixed paraffin-embedded primary DTC samples, using a commercial DNA extraction kit (GeneRead DNA formalin-fixed paraffin-embedded kit, catalog no. 180134; Qiagen) according to the manufacturer's instructions. The tumor samples were manually dissected under microscopic guidance by an empiric pathologist from Peking Union Medical College Hospital, and any adjacent normal tissue surrounding the tumor was pared.

\section{TERT Mutation Analysis}

TERT mutations were analyzed by polymerase chain reaction (PCR) amplification and direct Sanger sequencing of C228T and C250T, the hot mutation positions. A 193-bp fragment of TERT promoter was amplified by PCR on about $100 \mathrm{ng}$ of genomic DNA using primers 5'-CACCCGTCCTGCCCCTTCACCTT-3' (sense) and 5'-GGCTTCCCACGTGCGCAG CAGGA-3' (antisense). The thermal cycling conditions were as follows: initial denaturation for 3 min at $95^{\circ} \mathrm{C}, 40$ cycles of denaturation at $95^{\circ} \mathrm{C}$ for $30 \mathrm{~s}$, annealing at $68^{\circ} \mathrm{C}$ for $30 \mathrm{~s}$, elongation at $72^{\circ} \mathrm{C}$ for $30 \mathrm{~s}$, and final primer extension at $72^{\circ} \mathrm{C}$ for $5 \mathrm{~min}$. After quality confirmation by agarose gel electrophoresis, the PCR products were subjected to Sanger sequencing performed using an ABI Prism 3730 DNA Analyzer (Applied Biosystems).

\section{BRAF V600E Mutation Analysis}

Exon 15 of the BRAF gene containing the site for the T1799A (V600E) mutation was PCR-amplified using primers $5^{\prime}$-TGCTTGCTCTGATAGGAAAATG-3' (sense) and 5'-AGCCTCAATTCTTACCATCCA-3' (antisense). The PCR protocol comprised an initial denaturation for $3 \mathrm{~min}$ at $95^{\circ} \mathrm{C}, 40$ amplification cycles (denaturation for $30 \mathrm{~s}$ at $95^{\circ} \mathrm{C}$, annealing for $30 \mathrm{~s}$ at $60^{\circ} \mathrm{C}$, and extension for $30 \mathrm{~s}$ at $72^{\circ} \mathrm{C}$ ), and a final extension for $10 \mathrm{~min}$ at $72^{\circ} \mathrm{C}$. After quality confirmation by agarose gel electrophoresis, the PCR products were subjected to Sanger sequencing analysis as described above for TERT mutations.

\section{Statistical Analysis}

Statistical analyses were performed using the R Statistical Software Package (R Foundation for Statistical Computing). Continuous data are expressed as mean $\pm \mathrm{SD}$ and median. Fisher exact tests or $\chi^{2}$ tests were used to test for the significance of categoric data, and unpaired $t$ tests were used for continuous data. A $P$ value of less than 0.05 was considered to be statistically significant.

\section{RESULTS}

\section{Clinical and Molecular Characteristics}

Table 1 summarizes the clinical and molecular characteristics of the 66 distant metastatic DTC patients, 64 of whom had papillary thyroid cancer (PTC) and 2 follicular thyroid cancer. The femaleto-male ratio was $1.36: 1$, relatively lower than the DTC sex ratio reported previously (11). Only pulmonary metastasis was present in 59 patients, whereas 7 patients also had distant metastasis to other organs.

\section{Prevalence of TERT Mutation and BRAF Mutation in Distant Metastatic DTC}

The overall prevalence of TERT mutations was $22.73 \%$ (15/66), with the C228T mutation being more prevalent $(13 / 15,86.67 \%)$ than the C250T $(2 / 15,13.33 \%)$. Both TERT and BRAF mutations 
TABLE 1

Clinicopathologic Characteristics of the 66 DTC Patients with Distant Metastasis

\begin{tabular}{|c|c|c|c|}
\hline \multirow[b]{2}{*}{ Characteristic } & \multicolumn{2}{|c|}{ TERT } & \multirow[b]{2}{*}{$P$} \\
\hline & Mutation & Wild-type & \\
\hline Number of patients & 15 & 51 & \\
\hline Mean age $\pm S D(y)$ & $58.27 \pm 10.51$ & $36.86 \pm 13.98$ & $<0.001$ \\
\hline \multicolumn{4}{|l|}{ Sex } \\
\hline Female & 11 & 27 & 0.160 \\
\hline Male & 4 & 24 & \\
\hline Mean tumor diameter $\pm \mathrm{SD}(\mathrm{cm})$ & $4.12 \pm 2.38$ & $2.35 \pm 1.06$ & 0.013 \\
\hline \multicolumn{4}{|l|}{ Histologic diagnosis } \\
\hline Classic PTC & 6 & 18 & \\
\hline Follicular-variant PTC & 4 & 24 & \\
\hline Sclerosing diffuse PTC & 3 & 6 & \\
\hline Tall cell-variant PTC & 1 & 1 & \\
\hline Hobnail-variant PTC & 0 & 1 & \\
\hline Follicular thyroid cancer & 1 & 1 & \\
\hline Aggressive histologic subtypes & 5 & 9 & 0.344 \\
\hline Less aggressive histologic subtypes* & 10 & 42 & \\
\hline \multicolumn{4}{|l|}{ Multifocality } \\
\hline One lesion & 3 & 15 & 0.697 \\
\hline More than one lesion & 12 & 36 & \\
\hline \multicolumn{4}{|l|}{ Extrathyroidal invasion } \\
\hline Yes & 13 & 39 & 0.624 \\
\hline No & 2 & 12 & \\
\hline \multicolumn{4}{|l|}{ Lymph node metastasis } \\
\hline No & 0 & 2 & \\
\hline N1a & 0 & 0 & \\
\hline N1b & 12 & 44 & \\
\hline No cervical node dissection & 3 & 5 & \\
\hline \multicolumn{4}{|l|}{ Site of distant metastases } \\
\hline Lung & 12 & 47 & 0.386 \\
\hline Lung + other organs & 3 & 4 & \\
\hline \multicolumn{4}{|l|}{$B R A F$ mutation } \\
\hline Yes & 10 & 19 & 0.044 \\
\hline No & 5 & 32 & \\
\hline \multicolumn{4}{|l|}{ Refractory to radioiodine } \\
\hline Yes & 15 & 18 & $<0.001$ \\
\hline No & 0 & 33 & \\
\hline
\end{tabular}

${ }^{*}$ Classic PTC and follicular-variant PTC.

were found in 10 patients $(10 / 66,15.15 \%)$, with the other 5 patients $(7.58 \%)$ having TERT mutation alone. The BRAF mutation was found in 29 of the 66 patients $(43.94 \%$ ) (Fig.1; Tables 1 and 2).

\section{Association Between TERT Mutation and Clinicopathologic Characteristics}

TERT mutation was associated with older mean age at diagnosis $(t=5.480, P<0.001)$, larger mean tumor diameter $(t=2.803, P=$
0.013), and greater likelihood of both $B R A F$ mutation coexistence $\left(x^{2}=4.071, P=0.044\right)$ and refractoriness to radioiodine $\left(x^{2}=15.632, P<0.001\right)$ (Table 1$)$.

\section{Association Between TERT Mutation and Radioiodine Imaging Characteristics}

A significant association between TERT mutation and radioiodine uptake pattern was observed (Table 3). TERT mutation was associated with non-radioiodine-avid status. The T/B level on 


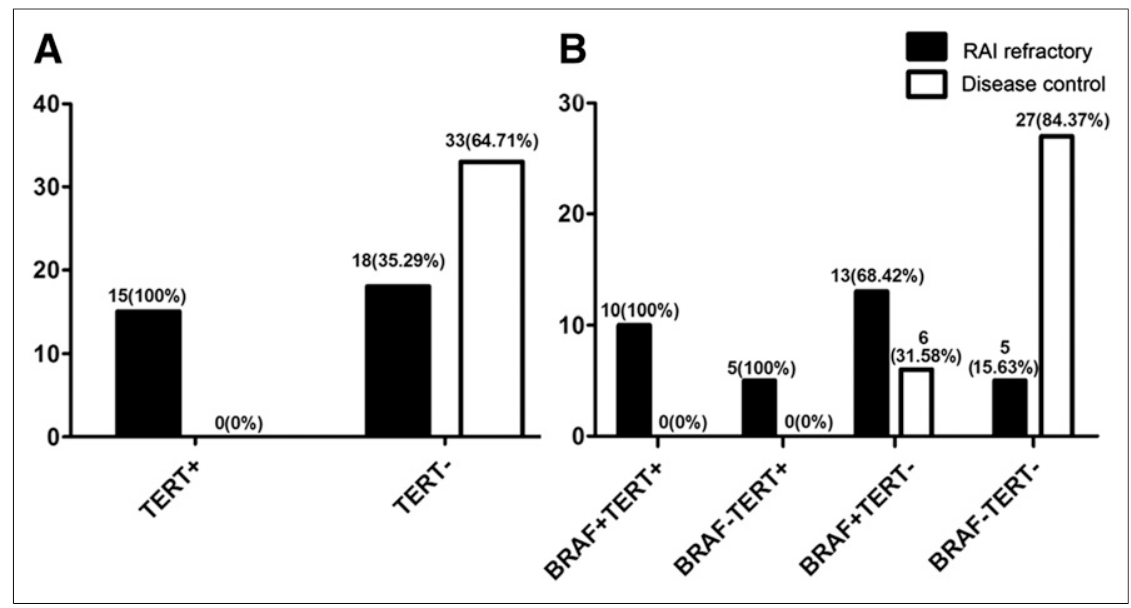

FIGURE 1. Association between TERT/BRAF mutation and radioiodine therapy response in distant metastatic DTC. (A) Association between TERT mutation and radioiodine therapy response (radioiodine-refractory or disease control). (B) Radioiodine responses of 4 different mutational scenarios. $\mathrm{RAI}=$ radioiodine.

whole-body scanning was much lower for TERT mutation than for TERT wild-type both after initial radioiodine therapy (2.04 vs. $18.54, P<0.001)$ and after subsequent radioiodine therapy when successful thyroid ablation was achieved (1.80 vs. $12.30, P<$ 0.001) (Table 3). Similarly, regardless of TERT mutation, BRAF mutation was also associated with non-radioiodine-avid status $(P<$ 0.05). Specifically, when TERT mutation coexisted with $B R A F$ mutation, the radioiodine uptake in metastatic foci was markedly reduced to near-background level, with T/B levels being significantly lower than for the $B R A F$-only group both after initial therapy (1.87 vs. $9.1, P=0.049)$ and when there was no remnant thyroid (1.61 vs.
7.43, $P=0.038$ ), indicating a complete dedifferentiation status (Table 3 ).

\section{Association Between TERT Mutation and Response to Radioiodine Therapy}

Figure 2 shows that serologic response differed significantly among the 4 mutational groups $(B R A F+T E R T+, \quad B R A F-T E R T+$, $B R A F+T E R T-$, and BRAF-TERT-, where $+=$ mutation and $-=$ wild-type; Fisher exact test, $P<0.05)$. A rising sTg level was seen in $93.33 \%(14 / 15)$ of the TERT mutation group, regardless of $B R A F$ mutation. None of the 15 TERT mutation patients showed a tendency toward decreasing $\mathrm{sTg}$. $B R A F$ mutation alone accounted for $52.63 \%$ of the patients with an sTg increase. Conversely, among cases negative for both mutations, $78.12 \%$ had an sTg decrease and only $12.50 \%$ had an sTg increase.

According to the criteria for radioiodine refractoriness, 33 cases were in the radioiodine-refractory group and the other 33 were in the diseasecontrol group. TERT mutation closely correlated with radioiodine refractoriness, with a high specificity of $100 \%$ and a positive predictive value of $100 \%$, suggesting a significant influence on a poor clinical outcome (Fig. 1A). The radioiodine responses of the 4 different mutational scenarios are shown in Figure 1B.

\section{Comparison of T/B Level Between BRAF Mutation Only and TERT Mutation Group}

Detailed clinical information was available for 36 patients, who underwent further T/B semiquantitative evaluation (Fig. 3; Table 3).

TABLE 2

Association Between Clinicopathologic Characteristics of DTC and BRAF Alone, TERT Mutation Alone, or Their Coexistence

\begin{tabular}{|c|c|c|c|c|}
\hline Characteristic & No mutation & TERT alone & $B R A F$ alone & $T E R T+B R A F$ \\
\hline Total DTC $(n=66)$ & 32 (48.48\%) & $5(7.58 \%)$ & $19(28.79 \%)$ & $10(15.15 \%)$ \\
\hline Age $\pm S D(y)$ & $35.00 \pm 14.73$ & $61.80 \pm 8.43$ & $40.00 \pm 12.34$ & $56.50 \pm 11.39$ \\
\hline \multicolumn{5}{|l|}{ Sex } \\
\hline Female & 15 & 4 & 12 & 7 \\
\hline Male & 17 & 1 & 7 & 3 \\
\hline \multicolumn{5}{|l|}{ Histologic diagnosis } \\
\hline Classic PTC & 11 & 2 & 7 & 4 \\
\hline Follicular-variant PTC & 16 & 1 & 8 & 3 \\
\hline Sclerosing diffuse PTC & 4 & 1 & 2 & 2 \\
\hline Tall cell-variant PTC & 0 & 0 & 1 & 1 \\
\hline Hobnail-variant PTC & 0 & 0 & 1 & 0 \\
\hline Follicular thyroid cancer & 1 & 1 & 0 & 0 \\
\hline \multicolumn{5}{|l|}{ TNM stage } \\
\hline II & 23 & 0 & 11 & 2 \\
\hline IV & 9 & 5 & 8 & 8 \\
\hline \multicolumn{5}{|l|}{ Refractory to radioiodine } \\
\hline Yes & 5 & 5 & 13 & 10 \\
\hline No & 27 & 0 & 6 & 0 \\
\hline
\end{tabular}


TABLE 3

T/B Level Measured After First Radioiodine Therapy and Successful Thyroid Ablation in Different Mutation Groups

\begin{tabular}{|c|c|c|c|c|}
\hline Parameter & T/B1 & $P$ & T/B2 & $P$ \\
\hline \multicolumn{5}{|c|}{ Irrespective of $B R A F$} \\
\hline TERT+ & $2.04 \pm 0.82$ & $<0.001$ & $1.80 \pm 0.97$ & $<0.001$ \\
\hline TERT- & $18.54 \pm 21.84$ & & $12.30 \pm 12.01$ & \\
\hline \multicolumn{5}{|c|}{ Irrespective of TERT } \\
\hline$B R A F+$ & $6.29 \pm 8.97$ & 0.012 & $5.17 \pm 6.86$ & 0.011 \\
\hline BRAF- & $23.45 \pm 24.92$ & & $14.76 \pm 13.19$ & \\
\hline \multicolumn{5}{|l|}{$B R A F+$} \\
\hline$B R A F+T E R T+$ & $1.87 \pm 0.72$ & 0.049 & $1.61 \pm 0.87$ & 0.038 \\
\hline$B R A F+T E R T-$ & $9.1 \pm 10.69$ & & $7.43 \pm 8.07$ & \\
\hline \multicolumn{5}{|l|}{$B R A F-$} \\
\hline BRAF-TERT+ & $3.22^{\star}$ & 0.420 & 3.13 & 0.380 \\
\hline BRAF-TERT- & $24.64 \pm 25.15$ & & $15.44 \pm 13.26$ & \\
\hline
\end{tabular}

${ }^{*} \mathrm{~T} / \mathrm{B}$ ratio was available for only one patient with TERT mutation alone.

$\mathrm{T} / \mathrm{B} 1$ = ratio after first radioiodine therapy, with thyroid remnant; $\mathrm{T} / \mathrm{B} 2$ = ratio after subsequent radioiodine therapy, without thyroid remnant.

T/B levels were compared between patients with TERT mutation and patients with $B R A F$ mutation only or no mutation. Compared with the group negative for both mutations, the TERT group had the lowest T/B level and the $B R A F$-only group had a relatively lower level. Interestingly, 8 patients with TERT mutation manifested as radioiodine-refractory even at the time of the first radioiodine therapy, and this status was confirmed at the time of the subsequent radioiodine therapy, when successful remnant ablation had been achieved. In the 11 patients with only BRAF mutation, $5(45.45 \%)$ exhibited non-radioiodine avidity initially and $2(18.18 \%)$ gradually lost the ability to trap radioiodine; thus, $7(63.64 \%)$ were eventually identified as radioiodine-refractory at the end of follow-up. Among the 17 patients negative for both mutations, although one (5.88\%) was non-radioiodine-avid initially and quickly became refractory to radioiodine treatment, the other $16(94.12 \%)$ manifested radioiodine accumulation and a good response to radioiodine treatment.

\section{Cases with Different TERT/BRAF Mutation Statuses and Radioiodine Therapy Responses}

Three representative cases based on the aforementioned scenarios are displayed in Figure 4. These show distinct correlations between different TERT/BRAF mutation statuses and radioiodine uptakes, as well as between different TERT/BRAF mutation statuses and radioiodine therapy responses.

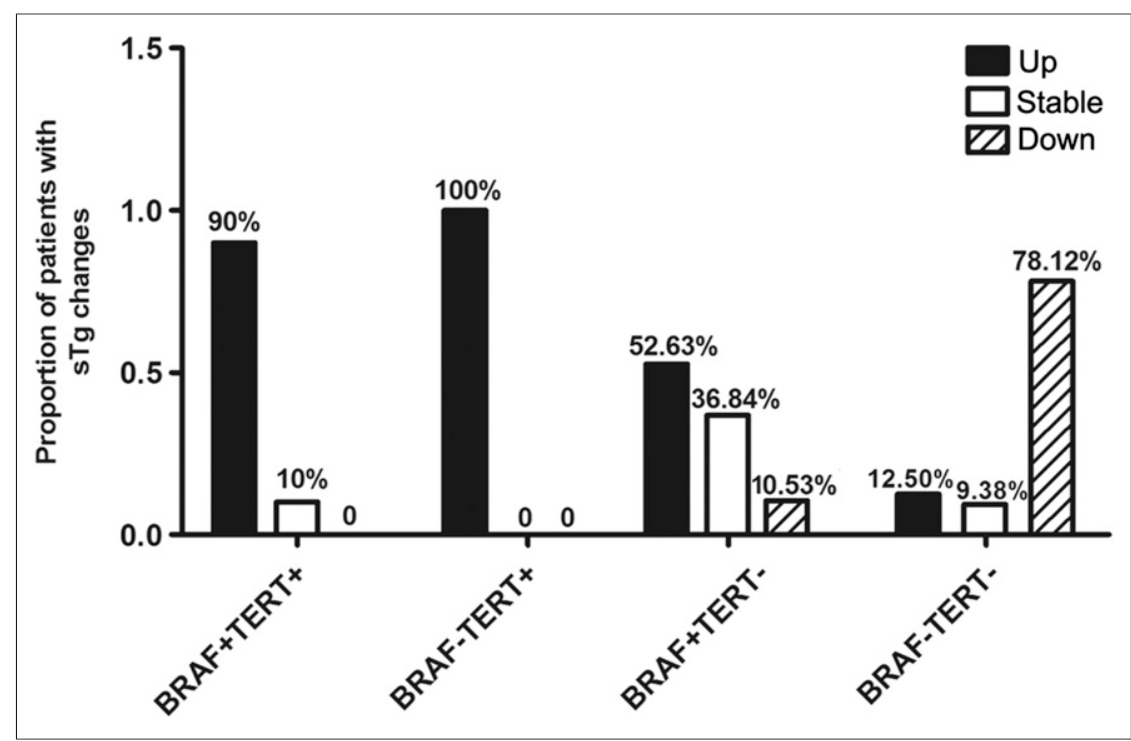

FIGURE 2. Serologic response to radioiodine therapy (up, stable, or down) of different mutational scenarios.

\section{DISCUSSION}

The prevalence of TERT mutation in aggressive thyroid cancer was first reported by Xing et al. (6) in 2014. Soon afterward, various studies demonstrated a consistent association between TERT mutation and poor clinicopathologic characteristics such as older age, the male sex, larger tumor size, extrathyroidal invasion, vascular invasion, distant metastasis, American Joint Committee on Cancer stage III or IV, recurrence, and death from follicular cellderived thyroid cancer (12-16). The poor prognosis of distant metastatic DTC is closely related to non-radioiodine avidity in distant metastatic lesions and eventually induces radioiodine-refractory status, thus posing a particularly tough prognostic and therapeutic challenge. Hence, early identification of lesions refractory to radioiodine could avoid repeated and time-consuming invalid application of radioiodine therapy 


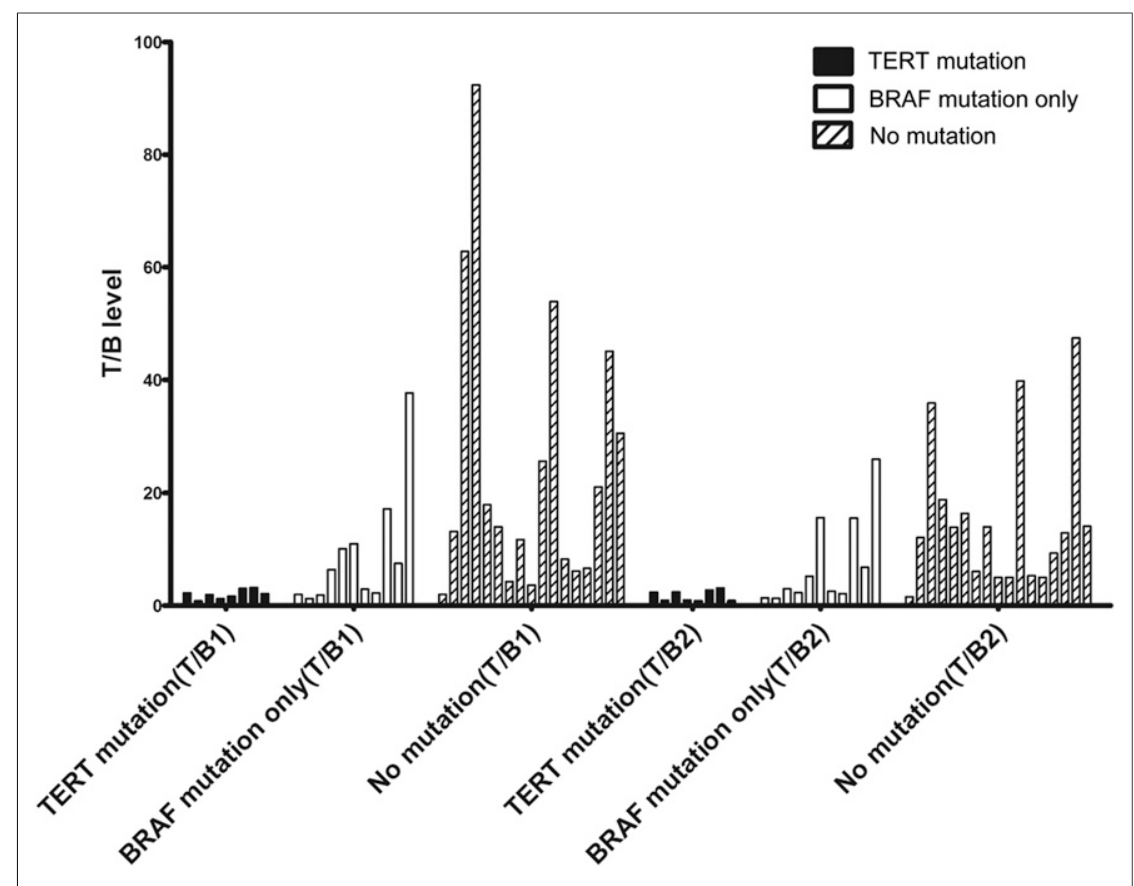

FIGURE 3. T/B levels of 36 cases of distant metastatic DTC. T/B1 $=$ ratio after first radioiodine therapy with thyroid remnant; T/B2 = ratio after subsequent radioiodine therapy without thyroid tissue remnant.

and earn more time for the use of alternative modalities. Molecular methods of identifying radioiodine-refractory lesions have been a subject of research. In our previous study, $B R A F$ mutation was shown to be a predictive molecular marker for non-radioiodine-avid status in distant metastatic DTC, thus outlining a novel molecular method of predicting lack of radioiodine avidity. Coexistence of TERT and BRAF mutation formed a genetic background that defined PTC with the worst clinical outcomes (14), yet it remains unclear how much TERT mutation may contribute to the prediction of non-radioiodine avidity and poor response to radioiodine therapy.

In this study, we found that the mean T/B level of radioiodine uptake in the TERT mutation group was significantly lower than that in the wild-type group. This result shows that TERT mutation holds a novel negative influence over radioiodine uptake by distant metastatic lesions. Several studies have found an association between TERT mutation and BRAF mutation and that coexistence of the two mutation types may trigger more aggressive clinicopathologic characteristics $(13,14,17-19)$. We have shown $B R A F$ mutation to be a predictor of non-radioiodine avidity. However, we observed different imaging patterns between BRAF mutation alone and TERT mutation, with a $100 \%$ non-radioiodine-avid presence in the TERT mutation group indicating that TERT mutation might hold a more potent negative influence over radioiodine uptake. The T/B levels also confirmed that coexistence of BRAF and TERT mutations may lead to the worst radioiodine uptake status, though a larger sample size is needed for further confirmation. Thus, for the first time, to our knowledge, we have demonstrated that TERT mutation is associated with both a molecular radioiodine imaging pattern and a non-radioiodine-avid status.

Several studies have demonstrated that non-radioiodine avidity is distinctly associated with a poor clinical outcome for patients with distant metastatic DTC $(3,20)$. For instance, Song et al. (20) reported that among patients with radioiodine-avid pulmonary metastases, $60.9 \%$ had significantly decreased thyroglobulin levels after radioiodine therapy and $60.3 \%$ had a reduction in metastases on follow-up CT. However, for non-radioiodine-avid patients, the survival rate has been seen to significantly decline $(3,20)$. A correlation has been found between clinical characteristics-including older age ( $>40 \mathrm{y}$ old), the combination of lung metastases with other distant metastases, and larger tumor size - and both radioiodine uptake and radioiodine therapy response (20). In this pioneering study, we suggested a novel molecular method of identifying both the radioiodine uptake status and the radioiodine therapy response of distant metastatic lesions. Our results show that TERT mutation can predict radioiodinerefractory disease with a positive predictive value of as high as $100 \%$, implying that TERT mutation is associated with both radioiodine uptake characteristics and clinical outcome. TERT mutation might be used as an early indicator of nonradioiodine-avid status and poor response to radioiodine therapy, thus allowing timely tailoring of management in these patients to other effective modalities, such as targeted therapy.

Because thyroglobulin plays an important role in response evaluation, we further evaluated the response of TERT mutation in terms of sTg through a biochemical sTg surveillance series. None of the 15 patients with TERT mutation tended to have an sTg decrease. By combining changes in sTg with radioiodine uptake status and other imaging evidence during follow-up, we could identify all TERT mutation patients as refractory to radioiodine therapy at the end of follow-up. It is still challenging for clinicians to judge the radioiodine refractory status early enough to individually tailor subsequent therapy. We have thus presented this striking relationship between TERT mutation and radioiodine therapy response, which might shed light on molecularly driven individual management in advanced thyroid cancer.

There have been studies showing that the prevalence of TERT mutation varies significantly among different countries, ranging from $4.1 \%$ to $25.5 \%$ for PTC (21-24) and from $5.9 \%$ to $36.4 \%$ for follicular thyroid cancer $(13,23)$. Among these data, the prevalence in China was reported to be $4.1 \%-11.3 \%$ for PTC $(13,21,24)$, relatively lower than in western countries. However, this observation remains limited because only 3 studies were conducted in China and no mutational data were documented on distant metastatic DTC, radioiodine concentrative characteristics, or radioiodine therapy response. Consistent with previous studies, the present study on Chinese patients found a TERT mutation prevalence of $22.73 \%(15 / 66)$ in patients with distant metastatic DTC, with C250T mutation also being less frequent $(2 / 15,13.33 \%)$. In contrast, the incidence of TERT mutation in patients with distant metastatic DTC is slightly more common in South Korea $(12 / 30,40 \%)$ and Italy $(14 / 43,33 \%)(15,25)$. Our data also showed that besides being 


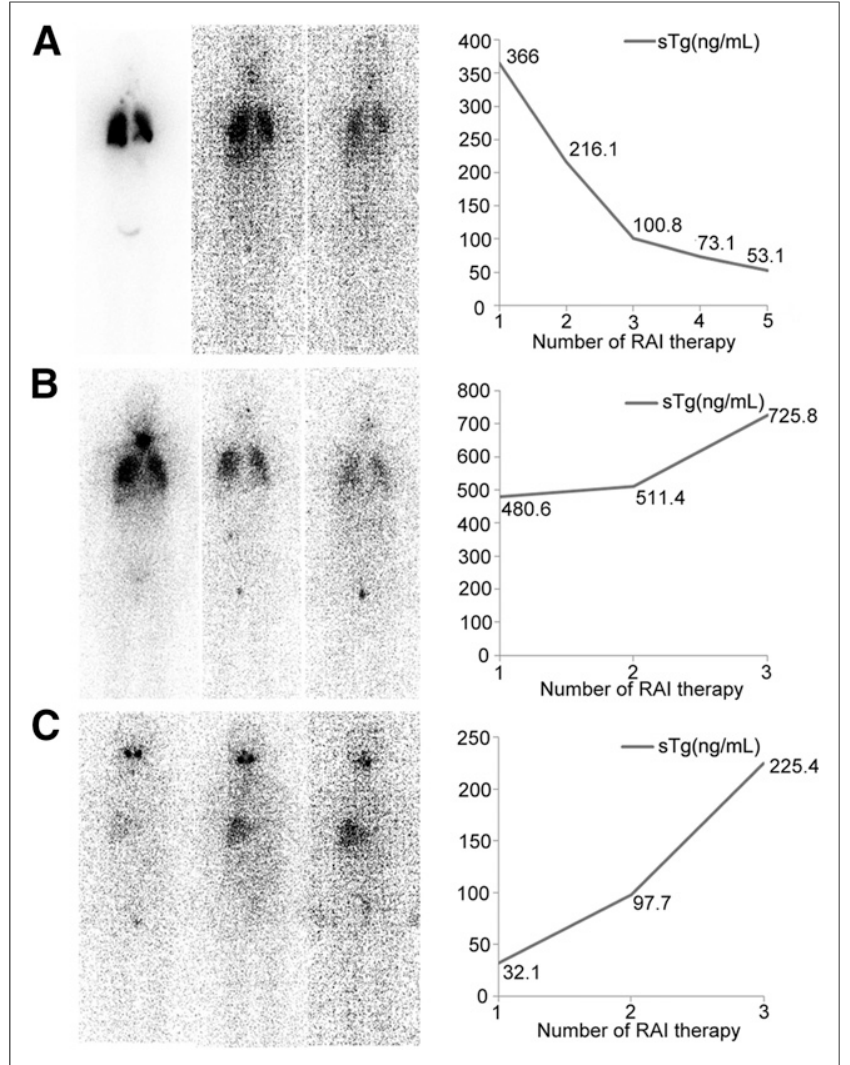

FIGURE 4. Three representative cases with different TERT/BRAF mutation statuses, radioiodine imaging results, and serologic responses. (A) A 53-y-old woman with pulmonary metastasis and negative for both mutations. Along with gradual decrease in radioiodine uptake by pulmonary metastatic foci after radioiodine therapies, simultaneous decline in sTg level was observed, indicating efficacy of therapy. (B) A 34-y-old woman with pulmonary metastasis and only $B R A F$ mutation. STg level increased gradually, albeit along with less accumulation of radioiodine by metastatic foci, implying a trend toward being refractory to radioiodine. (C) A 56-y-old woman with pulmonary metastasis and harboring both TERT and BRAF mutation. Lung metastatic foci never could accumulate radioiodine, and $\mathrm{sTg}$ level was sharply increased, indicating earlier inefficacy of radioiodine. $\mathrm{RAI}=$ radioiodine.

associated with radioiodine refractory status, TERT mutation in patients with distant metastatic DTC was associated with larger tumor size, older age, and greater likelihood of $B R A F$ mutation coexistence, in agreement with previously published data (15). These are factors also involved in the aggressive character of distant metastatic DTC, reminding us that apart from molecular properties, a multifactor mechanism might contribute to the progression of advanced thyroid cancer, especially radioiodinerefractory DTC.

Some limitations existed in this retrospective study. The cohort was relatively small because of the low incidence of distant metastasis in DTC, and the low prevalence of TERT mutation caused the subgroups to be even smaller, thus preventing further statistical analysis.

\section{CONCLUSION}

TERT promoter mutation is associated with non-radioiodine avidity in distant metastatic DTC and, compared with $B R A F$ mutation, has a greater negative influence on radioiodine uptake. TERT promoter mutation can be used as a novel molecular method of predicting radioiodine refractoriness

\section{DISCLOSURE}

This work was supported by grant 81571714 from the National Natural Science Foundation of China to Yansong Lin. No other potential conflict of interest relevant to this article was reported.

\section{ACKNOWLEDGMENTS}

We thank Chen Wang, Teng Zhang, and Xin Li for their help in collecting samples.

\section{REFERENCES}

1. Goffredo P, Sosa JA, Roman SA. Differentiated thyroid cancer presenting with distant metastases: a population analysis over two decades. World J Surg. 2013;37:1599-1605.

2. Haq M, Harmer C. Differentiated thyroid carcinoma with distant metastases at presentation: prognostic factors and outcome. Clin Endocrinol (Oxf). 2005;63:87-93.

3. Durante C, Haddy N, Baudin E, et al. Long-term outcome of 444 patients with distant metastases from papillary and follicular thyroid carcinoma: benefits and limits of radioiodine therapy. J Clin Endocrinol Metab. 2006; 91:2892-2899.

4. Zhao D, Jin X, Li F, Liang J, Lin Y. Integrin alphavbeta3 imaging of radioactive iodine-refractory thyroid cancer using ${ }^{99 m}$ Tc-3PRGD2. J Nucl Med. 2012;53:1872-1877.

5. Yang K, Wang H, Liang Z, Liang J, Li F, Lin Y. BRAFV600E mutation associated with non-radioiodine-avid status in distant metastatic papillary thyroid carcinoma. Clin Nucl Med. 2014;39:675-679.

6. Liu X, Bishop J, Shan Y, et al. Highly prevalent TERT promoter mutations in aggressive thyroid cancers. Endocr Relat Cancer. 2013;20:603-610.

7. Horn S, Figl A, Rachakonda PS, et al. TERT promoter mutations in familial and sporadic melanoma. Science. 2013;339:959-961.

8. Huang FW, Hodis E, Xu MJ, Kryukov GV, Chin L, Garraway LA. Highly recurrent TERT promoter mutations in human melanoma. Science. 2013;339:957959.

9. Haugen BR, Alexander EK, Bible KC, et al. 2015 American Thyroid Association management guidelines for adult patients with thyroid nodules and differentiated thyroid cancer: the American Thyroid Association guidelines task force on thyroid nodules and differentiated thyroid cancer. Thyroid. 2016;26:1-133.

10. Tan J, Jiang N, Li L, et al. ${ }^{131}$ I therapy guidelines for differentiated thyroid cancer (version 2014). Chin J Nucl Med Mol Imaging. 2014;34:264278.

11. Nilubol N, Zhang L, Kebebew E. Multivariate analysis of the relationship between male sex, disease-specific survival, and features of tumor aggressiveness in thyroid cancer of follicular cell origin. Thyroid. 2013;23:695702 .

12. Melo M, da Rocha AG, Vinagre J, et al. TERT promoter mutations are a major indicator of poor outcome in differentiated thyroid carcinomas. J Clin Endocrinol Metab. 2014;99:E754-E765.

13. Liu X, Qu S, Liu R, et al. TERT promoter mutations and their association with BRAF V600E mutation and aggressive clinicopathological characteristics of thyroid cancer. J Clin Endocrinol Metab. 2014;99:E1130-E1136.

14. Xing M, Liu R, Liu X, et al. BRAF V600E and TERT promoter mutations cooperatively identify the most aggressive papillary thyroid cancer with highest recurrence. J Clin Oncol. 2014;32:2718-2726.

15. Gandolfi G, Ragazzi M, Frasoldati A, Piana S, Ciarrocchi A, Sancisi V. TERT promoter mutations are associated with distant metastases in papillary thyroid carcinoma. Eur J Endocrinol. 2015;172:403-413.

16. Qasem E, Murugan AK, Al-Hindi H, et al. TERT promoter mutations in thyroid cancer: a report from a Middle Eastern population. Endocr Relat Cancer. 2015;22:901-908.

17. Ngeow J, Eng C. TERT and BRAF in thyroid cancer: teaming up for trouble. $J$ Clin Oncol. 2014;32:2683-2684. 
18. Melo M, da Rocha AG, Vinagre J, Sobrinho-Simoes M, Soares P. Coexistence of TERT promoter and BRAF mutations in papillary thyroid carcinoma: added value in patient prognosis? J Clin Oncol. 2015;33:667-668.

19. Dettmer MS, Schmitt A, Steinert H, et al. Tall cell papillary thyroid carcinoma: new diagnostic criteria and mutations in BRAF and TERT. Endocr Relat Cancer. 2015;22:419-429.

20. Song HJ, Qiu ZL, Shen CT, Wei WJ, Luo QY. Pulmonary metastases in differentiated thyroid cancer: efficacy of radioiodine therapy and prognostic factors. Eur J Endocrinol. 2015;173:399-408.

21. Jin L, Chen E, Dong S, et al. BRAF and TERT promoter mutations in the aggressiveness of papillary thyroid carcinoma: a study of 653 patients. Oncotarget. 2016;7:18346-18355.
22. Liu T, Wang N, Cao J, et al. The age- and shorter telomere-dependent TERT promoter mutation in follicular thyroid cell-derived carcinomas. Oncogene. 2014; 33:4978-4984.

23. Song YS, Lim JA, Park YJ. Mutation profile of well-differentiated thyroid cancer in Asians. Endocrinol Metab (Seoul). 2015;30:252-262.

24. Sun J, Zhang J, Lu J, et al. BRAF V600E and TERT promoter mutations in papillary thyroid carcinoma in Chinese patients. PLoS One. 2016;11: e0153319.

25. Bae JS, Kim Y, Jeon S, et al. Clinical utility of TERT promoter mutations and ALK rearrangement in thyroid cancer patients with a high prevalence of the BRAF V600E mutation. Diagn Pathol. 2016;11:21. 